\title{
TMD evolution as a double-scale evolution
}

\author{
Alexey Vladimirov* \\ Institut für Theoretische Physik, Universität Regensburg, D-93040, Regensburg, Germany \\ E-mail: alexey.vladimiroveur.de
}

Evolution of transverse momentum distributions (TMDs) is given by a pair of equations, which dictates the dependence on scales of rapidity and collinear factorizations. In this way, the evolution of TMDs takes a place in the two-dimension space of scales. It grants unique and non-trivial opportunities to simplify the task of TMD evolution and to disentangle perturbative series entering the TMD cross-section. In this talk, I present recent studies of the structure of double-scale evolution, the $\zeta$-prescription and the definition of optimal TMDs.

23rd International Spin Physics Symposium - SPIN2018 -

10-14 September, 2018

Ferrara, Italy

${ }^{*}$ Speaker. 


\section{Introduction}

The evolution procedure is the cornerstone of the factorization approach to the description of strong interactions. Each distribution or parameter depends on the renormalization scale and obeys the evolution equation. This is also the case of transverse momentum dependent distributions (TMDs), with the only difference that TMDs are dependent on two scales, and consequently, their evolution is given by a pair of equations. The double-scale dependence of TMD observables follows from the very structure of the factorization theorem at small transverse momentum, where one has to perform two successive factorization procedures each-of-which introduces a factorization scale. It is also the case of many multi-dimensional parton densities, such Transverse Momentum Dependent Distributions, jet-functions, double parton distributions, etc. In this talk, I present the recent study on the double-scale evolution performed in ref.[1]. To be more specific, I concentrate on the particular case of TMDs and their evolution, however, the approach and many conclusions can be easily applied to other double-scale objects, and extended to more involved multi-scale objects - subjects of multi-scale factorizations, see e.g. [2].

The double-scale evolution reveals certain problems and possibilities that are non-resolved in a single-scale case. There are two main of them. The first one is an issue. The usage of truncated perturbative series (fixed order or resumed) results to an ambiguity in the numerical realization of the solution. It is a well-known effect of the arbitrariness of the higher-then-given order terms in the solution of renormalization group equation. However, in the case of one-scale evolution, it does not violate the self-consistency of the solution, whereas in the double-evolution case it leads to the violation of transitivity rule. This ambiguity must be fixed in a particular way. Unfortunately, there are infinitely many ways to do so, and the selection of any corresponds to the selection of a particular model for unknown terms of perturbative series. I discuss the origin of this issue in sec.2, referring for an extended discussion to ref.[1]. The second one is an advantage. The double-scale evolution allows introducing the concept of equipotential (or null-evolution) lines in the space of scales. The value of TMDs is preserved at these lines, which leads to universal TMDs, that depends only on the "number" of equipotential line. It grants multiple simplifications in the theory development, as well as, in the practical implementation of TMD factorization. Originally, it has been introduced as $\zeta$-prescription in ref.[3], where it has been used for NNLO analysis for Drell-Yan data. Here, I discuss it in sec.3.

\section{TMD evolution factor in general form}

The evolution of TMDs (or TMD evolution for simplicity) is naturally formulated in the position space that is Fourier conjugated to the space of transverse momentum. It is given by the following pair of equations $[4,5,6,7]$

$$
\begin{aligned}
\mu^{2} \frac{d}{d \mu^{2}} F_{f}(x, b ; \mu, \zeta) & =\frac{\gamma_{F}^{f}(\mu, \zeta)}{2} F_{f}(x, b ; \mu, \zeta), \\
\zeta \frac{d}{d \zeta} F_{f}(x, b ; \mu, \zeta) & =-\mathscr{D}^{f}(\mu, b) F_{f}(x, b ; \mu, \zeta),
\end{aligned}
$$

where $F$ is a TMD distribution of the parton $f$ in hadron $h$. The argument $x$ is the usual Bjorken variable, and $b$ is the two-dimensional transverse distance. Importantly, the TMD evolution is the 
same for variety of TMDs, so it is the same for TMD parton distribution function (TMDPDF), and TMD fragmentation functions (TMDFF), as well as for TMDs of different polarizations. In fact, the only important quantum number of TMD evolution is the color representation of a parton $f$, which could be fundamental (for quarks) and adjoint (for gluons). The flavor of TMDs influences only the numerical values of anomalous dimension, and therefore, we omit it without loss of generality.

The pair of equations for TMD evolution corresponds to the two-step structure of TMD factorization. The first step is the ordinary hard-soft-collinear factorization, which is governed by the scale $\mu$. The second step is the factorization of rapidity divergences in the soft factor (proved recently at all orders in ref.[8]), which is governed by scale $\zeta$. The resulting TMD distributions are well-defined and could be used in phenomenology. The self-consistence of such definition has been checked perturbatively up to two-loop level for unpolarized [9], polarized [10] TMDPDF, as well as, for TMDFF [11]. The expressions for anomalous dimensions are known up to three-loop order $[12,13]$.

The system (2.1-2.2) is the system of ordinary differential equations those solution can be written as

$$
F\left(x, b ; \mu_{f}, \zeta_{f}\right)=R\left[b ;\left(\mu_{f}, \zeta_{f}\right) \rightarrow\left(\mu_{i}, \zeta_{i}\right)\right] F\left(x, b ; \mu_{i}, \zeta_{i}\right)
$$

where the function $R$ is called the TMD evolution factor. The pairs $\left(\mu_{f}, \zeta_{f}\right)$ and $\left(\mu_{i}, \zeta_{i}\right)$ refer respectively to a final and initial set of scales. In this way it is natural to think that TMD evolution takes a place in the two-dimension plane $(\mu, \zeta)$, see fig.1. Thus, the general expression for the evolution factor is

$$
R\left[b ;\left(\mu_{f}, \zeta_{f}\right) \rightarrow\left(\mu_{i}, \zeta_{i}\right)\right]=\exp \left[\int_{P}\left(\gamma_{F}(\mu, \zeta) \frac{d \mu}{\mu}-\mathscr{D}(\mu, b) \frac{d \zeta}{\zeta}\right)\right]
$$

where $\int_{P}$ denotes the line integral along the path $P$ in the $(\mu, \zeta)$-plane between initial and final points $\left(\mu_{f}, \zeta_{f}\right)$.

The uniqueness of solution (2.4) is guarantied by the integrability condition

$$
\zeta \frac{d}{d \zeta} \gamma_{F}(\mu, \zeta)=-\mu \frac{d}{d \mu} \mathscr{D}(\mu, b)
$$

This integrability condition is satisfied, due to the collinear overlap of ultraviolet and rapidity singularities, which result into relation between TMD anomalous dimensions and cusp-anomalous dimension

$$
\zeta \frac{d}{d \zeta} \gamma_{F}(\mu, \zeta)=-\Gamma(\mu), \quad \mu \frac{d}{d \mu} \mathscr{D}(\mu, b)=\Gamma(\mu)
$$

where $\Gamma$ is the (light-like) cusp anomalous dimension. Thanks to existence of integrability condition the integration path $P$ in (2.4) can be selected arbitrary path $P$.

For practical realization of TMD evolution factor, one set a particular choice of path $P$. The traditional choice, which could be traced to early works on Sudakov resummation [14], is the

$$
\text { path } 1:\left(\mu_{f}, \zeta_{f}\right) \rightarrow\left(\mu_{i}, \zeta_{f}\right) \rightarrow\left(\mu_{i}, \zeta_{i}\right)
$$


It is natural choice since the integration procedure becomes piecewise one-dimensional. And alternative path with similar properties is

$$
\text { path } 2:\left(\mu_{f}, \zeta_{f}\right) \rightarrow\left(\mu_{f}, \zeta_{i}\right) \rightarrow\left(\mu_{i}, \zeta_{i}\right) \text {. }
$$

In the $(\mu, \zeta)$-plane these paths form a rectangle, see fig. 1 . We call the solutions corresponding to these paths as solutions 1 and 2, for simplicity. Their explicit forms are

$$
\begin{array}{ll}
\text { solution 1: } & \ln R\left[b ;\left(\mu_{f}, \zeta_{f}\right) \stackrel{1}{\rightarrow}\left(\mu_{i}, \zeta_{i}\right)\right]=\int_{\mu_{i}}^{\mu_{f}} \frac{d \mu}{\mu} \gamma_{F}\left(\mu, \zeta_{f}\right)-\mathscr{D}\left(\mu_{i}, b\right) \ln \left(\frac{\zeta_{f}}{\zeta_{i}}\right), \\
\text { solution 2: } & \ln R\left[b ;\left(\mu_{f}, \zeta_{f}\right) \stackrel{2}{\rightarrow}\left(\mu_{i}, \zeta_{i}\right)\right]=\int_{\mu_{i}}^{\mu_{f}} \frac{d \mu}{\mu} \gamma_{F}\left(\mu, \zeta_{i}\right)-\mathscr{D}\left(\mu_{f}, b\right) \ln \left(\frac{\zeta_{f}}{\zeta_{i}}\right) .
\end{array}
$$

The solution 1 is widely used in the literature for TMD evolution (in fact, we can point only a few papers where it is not used). However, we stress that it is only a single (out of infinitely many) choice, which has not any special (apart of pure historical) background. In the following we present a better choices for paths based on the studies of structure of the TMD evolution plane.

Since the path of the evolution is unimportant the TMD evolution factor $R$ obeys the transitivity relation and inversion property

$$
\begin{aligned}
& R\left[b ;\left(\mu_{1}, \zeta_{1}\right) \rightarrow\left(\mu_{2}, \zeta_{2}\right)\right]=R\left[b ;\left(\mu_{1}, \zeta_{1}\right) \rightarrow\left(\mu_{3}, \zeta_{3}\right)\right] R\left[b ;\left(\mu_{3}, \zeta_{3}\right) \rightarrow\left(\mu_{2}, \zeta_{2}\right)\right], \\
& R\left[b ;\left(\mu_{1}, \zeta_{1}\right) \rightarrow\left(\mu_{2}, \zeta_{2}\right)\right]=R^{-1}\left[b ;\left(\mu_{2}, \zeta_{2}\right) \rightarrow\left(\mu_{1}, \zeta_{1}\right)\right],
\end{aligned}
$$

where $\left(\mu_{3}, \zeta_{3}\right)$ is arbitrary point in $(\mu, \zeta)$-plane. These equations are the cornerstones of the evolution mechanism, since they allow an universal definition of the non-perturbative distributions and the comparison of different experiments.

The serious drawback of perturbation theory is the violation of the integrability condition (2.5). One can check that at the given order of perturbation theory (say $a_{s}^{n}$ ) the integrability condition (2.5) is violated at next-to-given perturbative order $\left(a_{s}^{n+1}\right)$. Such a behavior is typical for truncated perturbative series, but in the case of TMD evolution, it results to a significant practical effect. Namely, the solution became dependent on the evolution path. Let us mention that paths 1 and 2 are inverse to each other, and therefore, the evolution forth and back with solution 1, in fact, uses different paths, and draw a rectangle in evolution plane, with area $S=\ln \left(\mu_{f}^{2} / \mu_{i}^{2}\right) \ln \left(\zeta_{f} / \zeta_{i}\right)$. Therefore, the numerical error in the application of solution 1 for evolution from initial-to-final-toinitial scales is of order $a_{s}^{n+1}$ times area $S$. For large scale separation, it could lead to tremendously sizable effect.

The path dependence of the solution is a rather unfortunate conclusion since it violates the transitivity and inversion rules (2.9-2.10). Moreover, the effect of violation (which is, formally, of the next-to-given perturbative order) is enchanted by large logarithm corrections, and by the area that presents the difference between paths. So, the typical effect of path dependence is $\sim L^{n+2} a_{s}^{n+1}$ (where $L$ is some a logarithm of typical scales). While the logarithmic corrections could be handled by resummations, which push the uncertainty down to $\sim L^{2} a_{s}^{n+1}$, the area correction could not be fixed by any perturbative method. Therefore, the effect of the inconsistency of TMD evolution became significant (with respect to accounted perturbative order) in two cases: the first one if the evolution spans a large range of energies (which implies large area factor), or at large-b (which 

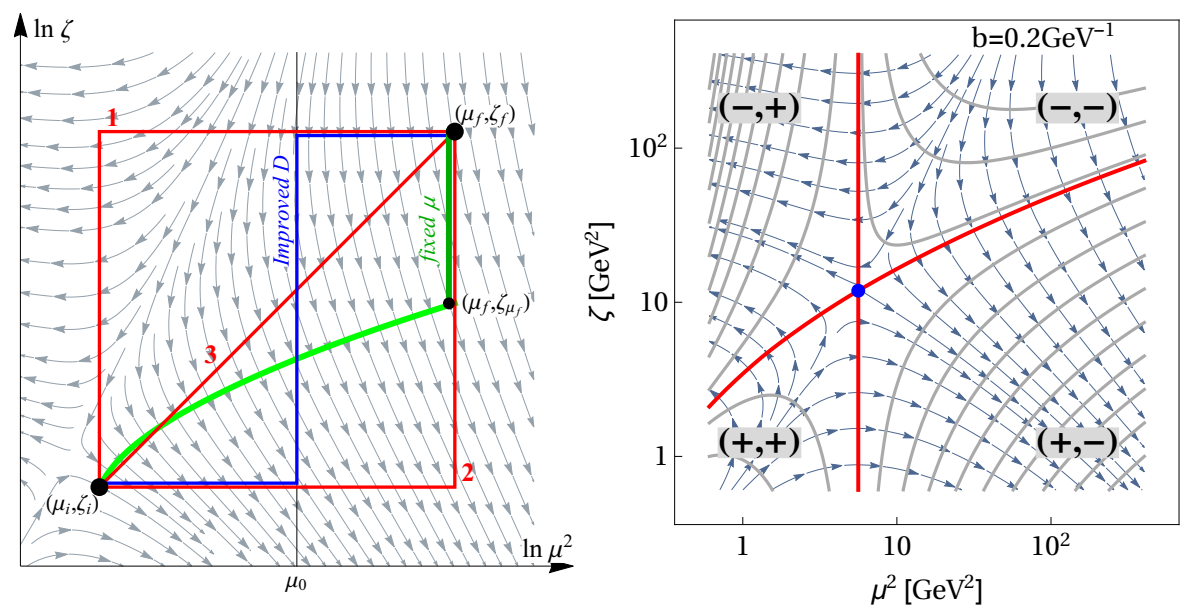

Figure 1: The evolution plane $(\mu, \zeta)$. The left panel demonstrate different choices for the evolution path, in particular paths 1 and 2 corresponds to solutions (2.7) and (2.8). The right panel demonstrates the singularity structure of the evolution potential, with gray lines being equipotential curves, red lines being special equipotential curves, and blue dot corresponds to the saddle point. The figures are taken from ref.[1], where descriptions of other elements can be found.

implies large logarithms at a fixed value of $a_{s}$ ). Let us stress that such situation happens not due to the presence of external uncontrolled parameter $b$, which is typically expected, but to a run of QCD coupling constant. I.e. in a conformal field theory the system of TMD equations is consistent at any given order of perturbation theory, which can be checked explicitly, see ref.[8].

In this talk, I do not present the solutions to this problem, and refer to the detailed discussion in ref.[1]. In what follows, I suppose that the problem of path-dependence is resolved in one or another way.

\section{Evolution potential and $\zeta$-prescription}

The character of evolution is dictated by the properties of anomalous dimensions, $\gamma_{F}$ and $\mathscr{D}$ in the plane $(\mu, \zeta)$. For the transparency I introduce the vector variable $\vec{v}$ for a point in $(\mu, \zeta)$ plane in logarithmic coordinates, $\vec{v}=\left(\ln \left(\mu^{2}\right), \ln (\zeta)\right)$. The pair of anomalous dimensions forms a vector field over $\vec{v}, \vec{E}(\vec{v}, b)=\left(\gamma_{F} / 2,-\mathscr{D}\right)$ (see fig.1 where this field is shown by arrows). In this notation the equations $(2.1,2.2)$ and their solution (2.4) are simply

$$
\vec{\nabla} F(x, b ; \vec{v})=\vec{E}(\vec{v}, b) F(x, b ; \vec{v}), \quad \ln R\left[b, \vec{v}_{f} \rightarrow \vec{v}_{i}\right]=\int_{P} \vec{E}(\vec{v}, b) \cdot d \vec{v},
$$

where $\nabla_{i}=d / d v_{i}$. Therefore, the evolution equations can be seen as a gradient equation for a two-dimensional system. The integrability condition (2.5) turns into

$$
\vec{\nabla} \times \vec{E}(\vec{v}, b)=0 .
$$

In other words the field $\vec{E}$ is irrotational, or conservative, and is given by a unique scalar potential

$$
\vec{E}(\vec{v}, b)=\vec{\nabla} U(\vec{v}, b) .
$$


The function $U$ is called evolution potential, and it solely determines the scaling behavior of TMDs. In particular, the evolution factor is given by a difference of potential between points

$$
\ln R\left[b, \vec{v}_{f} \rightarrow \vec{v}_{i}\right]=U\left(\vec{v}_{f}, b\right)-U\left(\vec{v}_{i}, b\right) .
$$

We note that the values of potential are dependent on the parameter $b$.

The equipotential lines play a special role in TMD evolution. Let us denote the equipotential curve which passes through the point $\vec{v}_{B}$ as $\vec{\omega}\left(\vec{v}_{B}, b\right)$, in fig.1(right) the equipotential lines are shown in gray. Along these curves, the scalar potential for TMD evolution does not change, and consequently, a TMD does not change its value with the change of scales along this line. It leads us to an important conclusion: the value of TMD distribution is dictated solely by an equipotential line to which it belongs. All TMDs belonging to the same curve are equivalent. Therefore, instead of studies of the evolution of TMDs with the scales $(\mu, \zeta)$ we should study its evolution with respect to "number" of the equipotential line $\rho$. In this way, the double-scale picture turns to a simpler single-scale picture, where the scale corresponds to a "number" of equipotential line. The evolution equation in the "number" space has usual form

$$
\frac{\partial F(x, b ; \rho)}{\partial \rho}=\frac{\partial U(\vec{v}(\rho), b)}{\partial \rho} F(x, b ; \rho) .
$$

The number $\rho$ enumerates the lines in some way, e.g. it could be a value of $\mu$ thought which the equipotential line passes at given $\zeta$.

The vector field $\vec{E}$ has several singularities in the finite domain of parameters. First, there is the line $\mu=\Lambda$ (where $\Lambda$ is the position of the Landau pole) at which both components of $\mathbf{E}$ turn to infinity. Second, there is a saddle point where both components of $\mathbf{E}$ turn to zero. In fig.1(right) the saddle point is depicted by a blue dot. The position of the saddle point is dictated by the equation

$$
\vec{E}\left(\vec{v}_{\text {saddle }}, b\right)=\overrightarrow{0} \text {. }
$$

The position of the saddle point depends on the parameter $b$ monotonously. It migrates to larger values of $(\mu, \zeta)$ with the decrease of $b$, and vice-versa. At some (large) value of $b$, the saddle point crosses the Landau pole line and escapes the observable region. The equipotential lines that pass through the saddle point dissect the evolution plane into quadrants. The evolution in each quadrant has its own particularities. Typically, the physically observable TMDs evolve in the first quadrant.

The equipotential line which passes through the saddle point we call the special equipotential line. Its particular values are dependent on a given set of anomalous dimensions, which, in turn, are dependent on the non-perturbative model and perturbative order. Nonetheless, for each set, the definition is unique and non-perturbative. The TMD distribution defined on the special equipotential line we call the optimal TMD distribution

$$
\text { optimal TMD distribution }=F\left(x, b ; \omega\left(\vec{v}_{\text {saddle }}(b)\right)\right) .
$$

This choice of the line has several important properties that justify its name. They are

- The definition of the special line is non-perturbative and unique, so the definition of optimal TMD. It provides simple way to compare various phenomenological models. All models must converge to a single value with the increase of perturbative accuracy and precision of the non-perturbative model. 
- Small-b operator product expansion is naively scale-invariant, which follows from the definition of TMDs on top of an equipotential line. The usage of the special equipotential line guarantees the absence of artificial singularities in the coefficient function and validity of the expression in the large domain of $b$. It follows from the simple fact that the special equipotential line is the only equipotential line that continuously spans the full range of $\mu$ and $\zeta$. This perturbative property of equipotential line has been first observed in ref.[3], and the corresponding formulation of TMD distributions was named $\zeta$-prescription.

- The optimal definition is independent of the perturbative order of evolution factor, and thus the evolution and the perturbative part of TMDs modeling are separated. In other words, the logarithms that appear within the small-b expansion of TMD are not related to logarithm in TMD evolution factor. It is not the case of other definitions, which define TMDs on lines non-equipotential lines. This is a very welcome feature since it allows to use the precise evolution factors (that is known to three-loop accuracy) together with tree-order and oneloop approximation for small-b expansion (which is the case of many polarized TMDs, for a review of recent status see ref.[15]), without theory tensions.

- Since special equipotential line passes through all values of $\mu$ at any value of $b$ (it is the feature of the special line only) one can use the simple path for the evolution factor. Namely, $\left(\mu_{f}, \zeta_{f}\right) \rightarrow\left(\mu_{f}, \zeta_{\mu_{f}}\right)$ where $\zeta_{\mu}$ belongs to $\omega$. Evolution along this path has fixed value of $\mu$ and shown in green in fig.1(left). For this path, the expression for evolution exponent is exceptionally simple

$$
R\left[b,\left(\mu_{f}, \zeta_{f}\right)\right]=\left(\frac{\zeta_{f}}{\zeta_{\mu_{f}}(b)}\right)^{-\mathscr{D}\left(\mu_{f}, b\right)}
$$

It contains no integrals and thus very fast numerically.

\section{Conclusion}

I have presented the main points of the recent study of double-scale evolution published in [1]. All these points are not specific to TMD distribution physics, but also applicable for all double-scale evolution, such as evolution of jet-functions, joint threshold resummation, and so on. The optimal definition of TMDs has been implemented in arTeMiDe package and used in the global analysis of TMD data. The results of this analysis demonstrate complete self-consistence and practicability of $\zeta$-prescription, and will be published soon.

\section{Acknowledgments}

I gratefully thank A. Bacchetta for invitation and support on SPIN2018.

\section{References}

[1] I. Scimemi and A. Vladimirov, JHEP 1808 (2018) 003 doi:10.1007/JHEP08(2018)003 [arXiv:1803.11089 [hep-ph]]. 
[2] I. W. Stewart, F. J. Tackmann and W. J. Waalewijn, Phys. Rev. D 81, 094035 (2010) doi:10.1103/PhysRevD.81.094035 [arXiv:0910.0467 [hep-ph]].

[3] I. Scimemi and A. Vladimirov, Eur. Phys. J. C 78 (2018) no.2, 89 doi:10.1140/epjc/s10052-018-5557-y [arXiv:1706.01473 [hep-ph]].

[4] J. Collins, Camb. Monogr. Part. Phys. Nucl. Phys. Cosmol. 32 (2011) 1.

[5] S. M. Aybat and T. C. Rogers, Phys. Rev. D 83 (2011) 114042 doi:10.1103/PhysRevD.83.114042 [arXiv:1101.5057 [hep-ph]].

[6] M. G. Echevarria, A. Idilbi and I. Scimemi, Phys. Lett. B 726 (2013) 795 doi:10.1016/j.physletb.2013.09.003 [arXiv:1211.1947 [hep-ph]].

[7] J. Y. Chiu, A. Jain, D. Neill and I. Z. Rothstein, JHEP 1205 (2012) 084 doi:10.1007/JHEP05(2012)084 [arXiv:1202.0814 [hep-ph]].

[8] A. Vladimirov, JHEP 1804 (2018) 045 doi:10.1007/JHEP04(2018)045 [arXiv:1707.07606 [hep-ph]].

[9] M. G. Echevarria, I. Scimemi and A. Vladimirov, JHEP 1609 (2016) 004 doi:10.1007/JHEP09(2016)004 [arXiv:1604.07869 [hep-ph]].

[10] D. Gutierrez-Reyes, I. Scimemi and A. Vladimirov, JHEP 1807 (2018) 172 doi:10.1007/JHEP07(2018)172 [arXiv:1805.07243 [hep-ph]].

[11] M. G. Echevarria, I. Scimemi and A. Vladimirov, Phys. Rev. D 93 (2016) no.1, 011502 Erratum: [Phys. Rev. D 94 (2016) no.9, 099904] doi:10.1103/PhysRevD.93.011502, 10.1103/PhysRevD.94.099904 [arXiv:1509.06392 [hep-ph]].

[12] A. A. Vladimirov, Phys. Rev. Lett. 118 (2017) no.6, 062001 doi:10.1103/PhysRevLett.118.062001 [arXiv:1610.05791 [hep-ph]].

[13] T. Gehrmann, E. W. N. Glover, T. Huber, N. Ikizlerli and C. Studerus, JHEP 1006 (2010) 094 doi:10.1007/JHEP06(2010)094 [arXiv:1004.3653 [hep-ph]].

[14] Y. L. Dokshitzer, D. Diakonov and S. I. Troian, Phys. Rept. 58 (1980) 269. doi:10.1016/0370-1573(80)90043-5

[15] I. Scimemi and A. Vladimirov, Eur. Phys. J. C 78 (2018) no.10, 802 doi:10.1140/epjc/s10052-018-6263-5 [arXiv:1804.08148 [hep-ph]]. 